\title{
Exploring the Impact of Task Density on Task Submission in Crowdsourcing Contests
}

\author{
Keng Yang ${ }^{*}$ \\ School of Information \\ Central University of Finance and Economics \\ Beijing, China \\ yangkengok@163.com
}

\author{
Baowen Sun \\ China Center for Internet Economy Research \\ Central University of Finance and Economics \\ Beijing, China \\ sunbaowen@263.net
}

\begin{abstract}
The competitive environment has a certain impact on the solvers' participation in the crowdsourcing contests. We Used the task density to represent the competitive environment, and the quantity of submitted solutions to represent the solvers' participation. Among them, the task density refers to the number of similar tasks in a certain period of time. The more similar tasks in a certain period of time, the greater the task density, indicating that there are more similar tasks to attract the solvers. Using the 2776 logo design task data on Tasken.com from the year 2015 to 2018, we analyzed the impact of task density on the solvers' participation and the regulating effect of the two important factors of task setting, namely task rewards and task duration, on the task density. The results showed that the task density was negatively correlated with the quantity of the task solutions submitted; the task duration was positively adjusting the impact of task density on the quantity of solution submitted, that is, increasing the task duration could reduce the negative impact of the competitive environment on the quantity of solutions submitted. The regulating effect of the task rewards on the relationship between the task density and the amount of solutions submitted was not significant. The possible reason is that increasing the rewards does not enable the solver to have more time to participate in other tasks. The task rewards have no regulating effect on the task density.
\end{abstract}

Keywords-Crowdsourcing contests; Task density; Task rewards; Task duration; Regulating effect

\section{INTRODUCTION}

Crowdsourcing activates today's technological revolution and unleashes the potential of everyone. The crowd is not only wise, talented and creative, but also productive [1]. A study found that disclosing information of pending issues to a large number of external solvers is an effective means of solving scientific problems. This approach solves the problem that one-third of large, well-known R\&D-intensive companies fail to solve in solving internal problems. Successful problem solving is related to the ability to attract professional respondents with multidisciplinary interests. Successful solutions transfer knowledge from one field to another, addressing boundary or external issues in its field of expertise [2].

In terms of organizational form, crowdsourcing is broadly divided into crowdsourcing contests [3] and non-competitive crowdsourcing $[4,5]$. Among them, one of the most popular and effective modes is the crowdsourcing contest. The typical performance of this model is that the task contractor publishes

National Key Research \& Development Plan of China: Research on Fundamental Theories and Methods of Crowd Science (2017YFB1400100)

National Natural Science Foundation of China: The study on the quality of users' ideas under crowdsourcing-based open innovation (71702206). the competition task through the crowdsourcing platform, the solvers selectively participate in and submit the solution, and the contractor selects the best solution and issues the rewards.

How to improve the quantity and quality of task solution submitted has become a hot topic in academia. A variety of factors affect the quantity of solutions submitted. Many scholars have studied the relationship between task rewards, task duration, difficulty levels of tasks, and the behavior of the solvers in the task setting process [6]. Not only the task type, task difficulty, task description, evaluation plan, task rewards, and task duration will affect the participation behavior of the solvers and thus the task performance, but also the credit of the contractor, the communication between the contractor and the receiver, and the competitive environment in which a task is located (such as task density), will also affect the solvers' participation and affect the final performance of the crowdsourcing contests. Generally, higher rewards, easier tasks, longer duration, and lower competitive intensity will attract more respondents [7]. A study to the crowdsourcing platform, Taskcn.com, found that task rewards are positively affecting the quantity and quality of task solutions submitted [8]. An analysis believes that although high monetary rewards can attract more solvers to compete, the probability of success for everyone will decrease because of the increased competition [9], while another analysis thinks that the additional effect of increased rewards can offset the reduction in the probability of winning due to intense competition [6]. A newer study also found that the intensity of competition is negatively correlated with the quantity of submissions, and the intensity of competition adjusts the relationship between task rewards and the quantity of submissions [10].

Existing research on the impact factors of crowdsourcing contests and solvers' participation mostly focused on analyzing task rewards, task duration and other factors, while there are few studies on competitive environment, such as task density. Research on task density mainly involves the relationship between task rewards, task density, and the quantity of solutions submitted. Regarding the role of regulation, existing studies have focused on the regulation of competition intensity on task rewards and submissions. we attempt to expand the existing research by incorporating task duration into the model, and using task rewards together as the model control variables. On this basis, we analyzed the impact of the task density on the quantity of task solutions submitted, 
and the regulating role of the task rewards and task duration on the task density.

\section{THEORETICAL BASIS}

The Expectancy-value theory proposed by Victor $\mathrm{H}$. Vroom in 1964 is part of the theory of behavioral motivational psychology [10] and is often used to explain the motivation of decision makers [11]. The decision maker will decide whether to complete a certain job according to the expected value of the behavior decision [12]. The more likely a task is to succeed, the greater the motivation for participating in it.

The expectancy-value theory holds that the degree of motivation of a target to a person is affected by two factors: one is the target valence, which refers to the subjective judgment of the person on how valuable it is to achieve the goal. If the goal is achieved, it will be of great value to people, and the enthusiasm of the person will be high; on the contrary, the enthusiasm will be low. The other is expectancy, which refers to the subjective estimate of the likelihood of a person achieving the goal. Only people think that the possibility of achieving this goal is very great, and they will strive to achieve it. If one thinks that the possibility of achieving this goal is small, or even impossible, the target incentive is small, and people will reduce or even not participate [13]. Later, people introduce instrumentality (which refers to non-personal factors that help individuals to achieve, such as the environment, shortcuts, task tools, etc.) into the expectancyvalue theory. For example, in an unfavorable environment, even if the valence and expectations are higher, the motivation of the person cannot be improved normally.

Expectation-value theory is widely used in crowdsourcing contests research. In the crowdsourcing contests, when the expected value of the solver's task rewards is higher, the incentive for the solver will increase, and the enthusiasm and effort of the solver to participate in the task will increase. If the expected value is lower, the enthusiasm of the solver to participate in the task will decrease. When the expected value is lower than the cost of the solver, the incentive level is minimized, and the number of respondents participating in the crowdsourcing contests will decrease [14]. Expectation-value theory suggests that people are more willing to accept tasks that are expected to be well-paid, and are not directly related to the actual amount of reward for a task. Therefore, what really motivates the crowdsourcing contests is the expected value of the reward after completing the task, rather than the actual amount of task rewards [15].

\section{DeVElopMent OF HyPOTHESES}

There is relatively little research on task density. Previous studies have analyzed the impact of task density on the solvers participation, the impact on the winning bid, and the impact of task density on the number of solvers. For example, Yang, et al. (2009) pointed out that some solvers are willing to participate in multiple tasks, which leads to insufficient efforts by the solvers to invest in a single solution task, and some solvers will choose the right task to answer according to their own abilities [16]. Therefore, for the same type of tasks, a higher task density will reduce the quantity of solution submissions for one of the tasks.
According to the expectancy-value theory, the solver chooses whether to participate in the task by the successful expected return value, and the successful expected return is more related to the winning probability [9]. Therefore, when the solver participates in multiple tasks at the same time and when the task density is large, the solver will make a choice based on the expected return and the probability of winning each task. For one task, the number of solutions may be reduced. Shao, et al. (2012) constructed a research model that influenced the quantity and quality factors of the contestant's arrangement attributes and market competition [7]. The model was tested using data from Taskcn.com and the result showed that task density affected the solvers participation. When task density was large, the solver could participate in any one or more competition tasks, the average number of solvers per task was reduced, and the degree of competition between solvers was reduced, thereby affecting the solver's winning bid.

It can be seen that when there are a large number of similar tasks in the same period, that is, when the task density is large, due to the limited energy, limited time and the different preferences of the solver, the possibility that the solver selects a similar task issued by one of the task contractors will be reduced, and then correspondingly the possibility of the quantity of solutions submitted will also be reduced. Thus, we propose the first hypothesis.

H1: Task density is negatively related to the quantity of task solutions submitted.

There are many studies on the impact of task rewards on the submission quantity of task solution. For example, Lakhani, et al. (2006) found that the main motivation of solvers is to get more money in crowdsourcing contests [2]. Reference [8] found that the higher the reward, the higher the number of participants in both the registration and submission stages. According to the expectancy-value theory, the solver regards the task rewards set by the task contractor as the expected value of participating in the crowdsourcing contests. Reference [17] found that the higher the task rewards, the higher the expected reward of the solver, and the more the task solution is submitted [17]. Reference [10] introduced the competitive intensity in the model, and the results showed that the intensity of competition has a negative impact on the relationship between task rewards and the quantity of solutions submitted. However, some scholars found that rewards have no significant impact on participation and submission, and may even play a role in reducing motivation for participation [18]. In order to analyze the reasons for the differences in previous studies, Yang and Sun, et al. (2018) based on the expectancy-value theory and self-efficacy evaluation theory, discussed the effect of task reward and duration on the quantity of solutions submitted by solvers, and found that there is tritely an inverted U-shaped effect of reward and duration on solvers' participation [19].

Since task rewards have a positive impact on the task submission quantity, when the positive impact is large enough, raising the task rewards will mitigate the negative impact of the task density on the quantity of submissions. In view of this, we proposed the second hypothesis. 
H2: Task rewards positively adjust the effect of task density on the quantity of task solutions submitted.

About the impact of task duration on the quantity of task solutions, plenty of earlier studies have shown that a longer duration will attract more solvers [20]. The longer the task duration, the more solvers can see the posted tasks, and the more solvers will participate, and the number of submissions will increase $[7,16]$. At the same time, most of the solvers use their spare time to participate in the crowdsourcing contests [2]. According to expectancy-value theory, for the solvers, the longer the task duration, the more time they will have to complete the task, and they can increase their expectations of gains by improving the quality of the task solution [21]. In contrast, some research showed that the longer the crowdsourcing contest duration was, the lower the working efficiency of solvers [22]. A recent study shows that task duration and the quantity of submissions is inverted U-shaped, task contractor need set proper task duration to attract more solvers, but not the longer the better. If the duration is longer than a threshold value, it will have negative effect on submissions [19].

Due to the positive impact of the task duration on the quantity of task solutions submitted, when the positive impact is large enough, extending the duration will reduce the negative impact of the task density on the quantity of submissions. Therefore, we proposed the third hypothesis.

H3: Task duration positively adjust the effect of task density on the quantity of task solutions submitted.

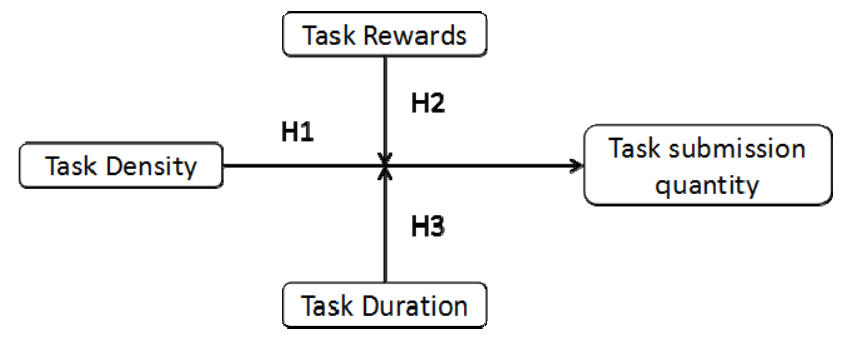

Fig. 1. Impact of task density on the quantity of the task solutions submitted.

\section{DATA AND MODEL}

The data in this paper is from Tasken.com, which is one of the biggest online crowdsourcing platforms in China and was founded in 2006, and the competition tasks involved include design, writing, and programming. As of February 2018, its registered users exceeded 3.68 million, tasks exceeded 63,000 and the total amount of tasks amounted to 40 million yuan (RMB). We used the web crawler tool written by JAVA program to capture all the tasks that had been publicized under the website reward task module, and a total of 3833 tasks completed between February 1, 2015 and January 1, 2018 were obtained. Among them, the logo design sub-class task is 2797, accounting for nearly 73 percent of the total number of tasks. After screening, 2776 pieces of valid data were obtained, all of which belong to the single-person winning mode. Descriptive statistics and correlation tests for the main variables are as follows.
Table II shows the descriptive statistics of the variables we used in our empirical analysis and Table III describes the correlations of variables.

TABLE I. VARIABLES DESCRIPTION

\begin{tabular}{|c|c|c|}
\hline $\begin{array}{l}\text { Variable } \\
\text { type }\end{array}$ & Variable name & Description \\
\hline $\begin{array}{l}\text { Dependent } \\
\text { Variable }\end{array}$ & $\begin{array}{l}\text { Quantity of } \\
\text { solutions } \\
\text { submitted (Y) }\end{array}$ & $\begin{array}{l}\text { The number of task solutions submitted } \\
\text { by the solvers }\end{array}$ \\
\hline \multirow[b]{3}{*}{$\begin{array}{l}\text { Independe } \\
\text { nt Variable }\end{array}$} & $\begin{array}{l}\text { Task intensity } \\
\text { (TD) }\end{array}$ & $\begin{array}{l}\text { The number of similar tasks in a certain } \\
\text { time range }\end{array}$ \\
\hline & Task rewards $(\mathrm{M})$ & $\begin{array}{l}\text { The monetary rewards that task } \\
\text { contractor set for the winner of the task }\end{array}$ \\
\hline & Task duration (D) & $\begin{array}{l}\text { The number of days between the release } \\
\text { of task and the end of the task, task } \\
\text { duration is set by task contractor and } \\
\text { used as measurement for task's time } \\
\text { limit }\end{array}$ \\
\hline \multirow{6}{*}{$\begin{array}{l}\text { Control } \\
\text { Variable }\end{array}$} & $\begin{array}{l}\text { Task description } \\
\text { length (TL) }\end{array}$ & $\begin{array}{l}\text { The number of words used in the task } \\
\text { description }\end{array}$ \\
\hline & $\begin{array}{l}\text { The rask } \\
\text { contractor's } \\
\text { (Credit) }\end{array}$ & $\begin{array}{l}\text { The task contractor's credit in } \\
\text { Taskcn.com }\end{array}$ \\
\hline & $\begin{array}{l}\text { The task } \\
\text { contractor's } \\
\text { experience (Exp) }\end{array}$ & $\begin{array}{l}\text { The number of tasks that the task } \\
\text { contractor has released in Taskcn.com }\end{array}$ \\
\hline & $\begin{array}{l}\text { Satisfaction rate } \\
\text { (Rate) }\end{array}$ & $\begin{array}{l}\text { The favorable rate received by the task } \\
\text { contractor in Taskcn.com }\end{array}$ \\
\hline & $\begin{array}{l}\text { Real name } \\
\text { authentication } \\
\text { (Real) }\end{array}$ & $\begin{array}{l}\text { Real-name certification of the task } \\
\text { contractor. Set it as a dummy variable. } \\
\text { When it is 1, it indicates that } \\
\text { authentication is performed, and } 0 \\
\text { indicates that it is not authenticated. }\end{array}$ \\
\hline & $\begin{array}{l}\text { Email } \\
\text { authentication } \\
\text { (Email) }\end{array}$ & $\begin{array}{l}\text { The mailbox of the task contractor is } \\
\text { authenticated. Set it as a dummy } \\
\text { variable. When it is } 1 \text {, it indicates that } \\
\text { the authentication is performed, and if it } \\
\text { is } 0 \text {, it is not authenticated. }\end{array}$ \\
\hline
\end{tabular}

a. Note: all the control variables will affect the quantity of task solutions submitted, so it is necessary to include them in the model.

TABLE II. DESCRIPTIVE STATISTIC

\begin{tabular}{|l|l|l|l|l|l|}
\hline \multicolumn{1}{|c|}{ stats } & \multicolumn{1}{c|}{ Y } & \multicolumn{1}{c|}{ M } & \multicolumn{1}{c|}{ D } & \multicolumn{1}{c|}{ TD } & \multicolumn{1}{c|}{ TL } \\
\hline mean & 28.36 & 525.5 & 37.86 & 16.57 & 217.9 \\
\hline sd & 21.67 & 690.1 & 97.03 & 5.712 & 322.5 \\
\hline p50 & 23 & 300 & 18.07 & 17 & 63 \\
\hline $\min$ & 1 & 100 & 0.190 & 0 & 0 \\
\hline $\max$ & 171 & 20000 & 1274 & 35 & 2052 \\
\hline N & 2776 & 2776 & 2776 & 2776 & 2776 \\
\hline stats & \multicolumn{1}{|c|}{ Rate } & Email & Real & Credit & \multicolumn{1}{|c|}{ Exp } \\
\hline mean & 0.999 & 0.468 & 0.162 & 15.91 & 4.003 \\
\hline sd & 0.0223 & 0.499 & 0.369 & 24.54 & 6.671 \\
\hline p50 & 1 & 0 & 0 & 7 & 2 \\
\hline min & 0 & 0 & 0 & 0 & 1 \\
\hline max & 1 & 1 & 1 & 210 & 67 \\
\hline $\mathrm{N}$ & 2751 & 2776 & 2776 & 2774 & 2772 \\
\hline
\end{tabular}

The model for task density versus the quantity of task solution submitted is as follows (1):

$$
\begin{aligned}
& \ln \mathrm{Y}=\mathrm{a}_{0}+\mathrm{a}_{1} \ln T D+a_{2} \ln M+a_{3} \ln d+a_{4} \ln T D^{*} \ln D \\
& +a_{4} \ln T D^{*} \ln M+\gamma X+\mu_{0}
\end{aligned}
$$


TABLE III. DESCRIPTIVE STATISTICS

\begin{tabular}{|c|c|c|c|c|c|c|c|c|c|c|}
\hline & $\ln Y$ & $\ln M$ & $\ln D$ & $\ln T D$ & TL & Credit & Exp & Rate & Email & Real \\
\hline $\ln Y$ & 1 & & & & & & & & & \\
\hline $\ln M$ & $0.5129 *$ & 1 & & & & & & & & \\
\hline $\operatorname{lnD}$ & $0.3315^{*}$ & $0.2155^{*}$ & 1 & & & & & & & \\
\hline $\operatorname{lnTD}$ & $-0.0513^{*}$ & 0.00430 & $-0.1421 *$ & 1 & & & & & & \\
\hline TL & $0.0690^{*}$ & 0.1937* & $0.0982 *$ & -0.0661* & 1 & & & & & \\
\hline Credit & $0.1566^{*}$ & $0.3661^{*}$ & $0.1550^{*}$ & -0.0133 & $0.1027^{*}$ & 1 & & & & \\
\hline Exp & $-0.0709 *$ & $-0.1083 *$ & $0.1503^{*}$ & -0.0350 & 0.0191 & $0.6131^{*}$ & 1 & & & \\
\hline Rate & 0.0285 & 0.0165 & 0.0131 & -0.00420 & 0.00440 & 0.0160 & 0.00980 & 1 & & \\
\hline Email & 0.00530 & 0.0160 & 0.0451 & -0.0110 & 0.0435 & $0.1759^{*}$ & 0.2014* & 0.0286 & 1 & \\
\hline Real & 0.00790 & 0.0339 & 0.000800 & 0.00860 & 0.0434 & $0.2237^{*}$ & $0.1498^{*}$ & 0.00680 & $0.3734^{*}$ & 1 \\
\hline
\end{tabular}

In the model processing, we centralized $\mathrm{TD}, \mathrm{M}$, and $\mathrm{D}$ to eliminate the effects of multi-collinearity. $X$ represents the control variable group.

\section{EMPIRICAL RESULTS}

We used the OLS method to regress the model and the specific empirical results are shown in the table IV.

TABLE IV. EMPIRICAL RESULTS

\begin{tabular}{|c|c|c|c|c|}
\hline & (1) & (2) & (3) & (4) \\
\hline & $\ln Y$ & $\ln Y$ & $\ln Y$ & $\ln Y$ \\
\hline \multirow{2}{*}{$\begin{array}{l}\operatorname{lnTD} \\
\text { (Not decentralized) }\end{array}$} & -0.1865 & -0.1943 & & \\
\hline & $(0.366)$ & $(0.346)$ & & \\
\hline \multirow[t]{2}{*}{$\ln M$} & $0.4735^{* * *}$ & $0.4907^{* * *}$ & & \\
\hline & $(0.000)$ & $(0.000)$ & & \\
\hline \multirow[t]{2}{*}{$\ln \mathrm{D}$} & -0.0113 & -0.0025 & & \\
\hline & $(0.806)$ & $(0.956)$ & & \\
\hline \multirow[t]{2}{*}{ interlnTD_M } & -0.0212 & -0.0207 & & \\
\hline & $(0.532)$ & $(0.543)$ & & \\
\hline \multirow[t]{2}{*}{ interlnTD_D } & $0.0809^{* * *}$ & $0.0805^{* * *}$ & & \\
\hline & $(0.000)$ & $(0.000)$ & & \\
\hline \multirow{2}{*}{$\begin{array}{l}\text { c_lnTD } \\
\text { (Decentralized) }\end{array}$} & & & $-0.0763^{*}$ & $-0.0819^{* * *}$ \\
\hline & & & $(0.010)$ & $(0.006)$ \\
\hline \multirow[t]{2}{*}{ c_lnM } & & & $0.4153^{* * *}$ & $0.4340^{* * *}$ \\
\hline & & & $(0.000)$ & $(0.000)$ \\
\hline \multirow[t]{2}{*}{ c_ln $\ln$} & & & $0.2104^{* * *}$ & $0.2180^{* * * *}$ \\
\hline & & & $(0.000)$ & $(0.000)$ \\
\hline \multirow{2}{*}{ inter_lnTD_M } & & & -0.0212 & -0.0207 \\
\hline & & & $(0.532)$ & $(0.543)$ \\
\hline \multirow[t]{2}{*}{ inter_lnTD_D } & & & $0.0809^{* * *}$ & $0.0805^{* * *}$ \\
\hline & & & $\begin{array}{l}(0.000) \\
\end{array}$ & $(0.000)$ \\
\hline Control X & $\mathrm{N}$ & $\mathrm{Y}$ & $\mathrm{N}$ & $\mathrm{Y}$ \\
\hline \multirow[t]{2}{*}{ cons } & 0.5441 & -0.1747 & $3.0807^{* * *}$ & $2.4725^{* * *}$ \\
\hline & $(0.343)$ & $(0.826)$ & $(0.000)$ & $\begin{array}{l}(0.000) \\
\end{array}$ \\
\hline $\mathrm{N}$ & 2753 & 2724 & 2753 & 2724 \\
\hline $\mathrm{R}^{2}$ & 0.331 & 0.337 & 0.331 & 0.337 \\
\hline $\mathrm{F}$ & 271.5431 & 125.3917 & 271.5431 & 125.3917 \\
\hline
\end{tabular}

c. Note: P-values in parentheses, * means $\mathrm{p}<0.05, * *$ means $\mathrm{p}<0.01,{ }^{* * *}$ means $\mathrm{p}<0.001$; Control X indicates whether the control variable is controlled, $\mathrm{Y}$ means control variables are included, $\mathrm{N}$ means control variables not included. b. Note: The asterisk $(*)$ indicates that the correlation coefficient is significant at a confidence level of 0.01 .

Table IV shows the regression results before and after the decentralization of the sample data and before and after the introduction of the control variables. It can be seen that the regression coefficients of the results in columns 1 and 2 of the table are not significant, which may be due to the multicollinearity of task density and task duration and task rewards. This can be seen by analyzing the correlation coefficients of the variables. Columns 3 and 4 of the table are regression results of the data decentralized, and the coefficients are significant. Therefore, the regression results of columns 3 and 4 are mainly selected in this paper.

We can see that task density has a significant negative relationship with the quantity of task solutions submitted, and $\mathrm{H} 1$ is verified. This shows that the higher the task density, the lower the quantity of solutions submitted per task. The interaction term coefficient of the task duration and the task density is positive, indicating that task duration makes the negative relationship between task density and the quantity of solutions submitted smaller. That is, as task duration increases, the negative effect of the task density on the quantity of solutions submitted decreases. H3 is verified.

However, for task rewards, the adjustment effect of task rewards on the relationship between task density and the quantity of solutions submitted is not significant. H2 does not hold. The possible reasons are as follows: the task density mainly reflects the number of similar tasks in a certain time, the time required for solvers to participate in a task is relatively unchanged, many solvers participate in Taskcn.com in their spare time and increasing rewards does not give solvers more time to participate in other projects.

\section{CONCLUSIONS}

We got three conclusions. First, task density has a negative relationship with the quantity of the task solutions submitted; Second, increasing task rewards does not give solvers more time to participate in other projects, and the regulating effect of task rewards on the relationship between task density and the quantity of the task solutions submitted is not significant; Third, task duration positively adjusts the impact of task density on the quantity of solutions, and increasing task duration can mitigate the negative impact of task density on the quantity of the task solutions.

From the perspective of the task contractor, we analyzed the influence mechanism of objective competition 
environment on the solvers participation in the crowdsourcing contests. We not only analyzed the impact of task density on the quantity of the task solutions that solvers submitted, but also incorporating task rewards into the model, more importantly introduced task duration. In addition, we further analyzed the regulating effect of the task rewards and task duration on the quantity of task solutions. To a certain degree, our analysis has deepened the research content of task performance in crowdsourcing contests. At the same time, from the task contractor's point of view, adding rewards is equivalent to directly increasing the cost of the task, which is not a good means. Yet, by appropriately increasing the task duration, more solvers' working hours will be matched to the task, and it will result in more solutions and more submissions. This shows that our analysis also has certain practical reference value for the task setting and release of the task contractor.

Although we expected that the task rewards can positively regulate the impact of the task density on the quantity of submitted solutions. But the empirical results did not support this expectation. In addition to the possible reasons mentioned above, whether there are other factors that contribute to this result requires further research. Moreover, we only analyzed the impact of task density on the quantity of task solutions, and did not analyze its impact on the quality of task solutions. Higher task density reduces the quantity of submissions, but is it possible to improve the quality of the solutions? This also requires further research.

\section{REFERENCES}

[1] J. Howe, Crowdsourcing: Why the Power of the Crowd Is Driving the Future of Business, Crown Publishing Group, 2010.

[2] K.R. Lakhani, L.B. Jeppesen, P.A. Lohse, and J.A. Panetta, “ The value of openness in scientific problem solving," Harvard Business Review, Working Paper No.07-050,January 2006.

[3] K.J. Boudreau, N. Lacetera, and K.R. Lakhani, "Incentives and problem uncertainty in innovation contests: an empirical analysis," Management Science, vol. 57(5), pp. 843-863, 2011.

[4] D.G.P. Micheal, The co-creation of value: exploring engagement behaviors in user-generated content websites. Dissertations \& Theses Gradworks, 2010.

[5] B.L. Bayus, "Crowdsourcing new product ideas over time: an analysis of the dell ideastorm community," Management Science, vol. 59(1), pp. 226-244, 2013.

[6] Y. Huang, P.V. Singh, and T. Mukhopadhyay, "How to Design Crowdsourcing Contest: A Structural Empirical Analysis," Conference on Information Systems and Technology (CIST 2012), Phoenix, Arizona 2012, pp. 1-18.
[7] B. Shao, L. Shi, B. Xu, and L. Liu, "Factors affecting participation of solvers in crowdsourcing: an empirical study from china," Electronic Markets, vol. 22(2), pp. 73-82, 2012.

[8] T.X. Liu, J. Yang, L.A. Adamic, and Y. Chen, "Crowdsourcing with allpay auctions: a field experiment on taskcn," Proceedings of the American Society for Information Science \& Technology, vol. 48(1), pp. $1-4,2012$.

[9] G. Amihai and H. Refael, "Optimal Contests," Economic Inquiry, vol 26(1), pp. 133-143, 1988.

[10] D. Li and L. Hu, "Exploring the effects of reward and competition intensity on participation in crowdsourcing contests," Electronic Markets, vol. 27(3), pp. 199-210, 2017.

[11] R.H. Rasch and H.L. Tosi, "Factors affecting software developers" performance: an integrated approach," Mis Quarterly, vol. 16(3), pp. 395-413, 1992.

[12] H.A. Murray, "Explorations in personality: a clinical and experimental study of fifty men of college age," American Journal of Sociology, vol. 4(4), pp. 576-583, 1938.

[13] V.H. Vroom, "Work and motivation," Industrial Organization Theory \& Practice, vol. 35(2), pp. 2-33, 1964.

[14] J. Shah and E.T. Higgins, "Expectancy x value effects: regulatory focus as determinant of magnitude and direction," J Pers Soc Psychol, vol. 73(3), pp. 447-458, 1997.

[15] A. Wigfield, S. Tonks, and S.L. Klauda, Expectancy-value theory. Handbook of motivation at school, 2016, pp. 55-74.

[16] Y. Yang, P.Y. Chen, and P.A. Pavlou, "Open Innovation: An Empirical Study of Online Contests," International Conference on Information Systems, Icis 2009, Phoenix, Arizona, USA, 2009, pp. 13.

[17] Y. Sun, N. Wang, C. Yin, and J.X. Zhang, "Understanding the relationships between motivators and effort in crowdsourcing marketplaces: a nonlinear analysis," International Journal of Information Management, vol. 35(3), pp. 267-276, 2015.

[18] J. Yang, L.A. Adamic, and M.S. Ackerman, "Competing to Share Expertise: The Tasken Knowledge Sharing Community," International Conference on Weblogs and Social Media, Icwsm 2008, Seattle, Washington, Usa, 2008.

[19] K. Yang, B.W. Sun, Q. Liu, and W.G. Fan, "Understanding the Effects of Task Reward and Duration on Solvers Participation in Crowdsourcing Contests," Paper presented at the CSWIM2018, Qingdao, China, 2018, pp. 464-470.

[20] D. Gefen, G. Gefen, and E. Carmel, "How project description length and expected duration affect bidding and project success in crowdsourcing software development," Journal of Systems \& Software, vol. 116, pp. 75-84, 2016.

[21] J.J. Horton and L.B. Chilton, "The labor economics of paid crowdsourcing," Proceedings of the 11th ACM conference on Electronic commerce, Cambridge, Massachusetts, USA, 2010, pp. 209-218.

[22] C. Terwiesch and Y. Xu, "Innovation contests, open innovation, and multiagent problem solving," Management Science, vol. 54(9), pp. 1529-1543, 2008. 